\title{
Geometry Modulation of Microlens Array Using Spin Coating and Evaporation Processes of Photoresist Mixture
}

\author{
Suho Kim ${ }^{1,2}$, Eunju Yeo', Jeong Hwan Kim ${ }^{1,3}$, Yeong-Eun Yoo ${ }^{1,3}$, Doo-Sun Choi ${ }^{1,3}$, and Jae Sung Yoon ${ }^{1,3,1}$ \\ 1 Department of Nano Manufacturing Technology, Korea Institute of Machinery and Materials, 156, Gajeongbuk-ro, Yuseong-gu, Daejeon, 305-343, South Korea \\ 2 Department of Mechanical Engineering, Chungnam National University, 99, Daehak-ro, Yuseong-gu, Daejeon, 305-764, South Korea \\ 3 Department of Nano-mechatronics, Korea University of Science and Technology, 217, Gajeong-ro, Yuseong-gu, Daejeon, 305-350, South Korea \\ \# Corresponding Author / E-mail: jaesyoon@kimm.re.kr, TEL: +82-42-868-7617, FAX: +82-42-868-7149
}

KEYWORDS: Microlens array, Photoresist, Surface tension, Spin coating, Volume shrinkage

\begin{abstract}
Recently, cost effectiveness and environmental consideration have become essential issues in the fabrication techniques. In this study, microlens array has been fabricated with simple processes. On a silicon substrate, which has circular micro holes, photoresist mixture has been coated using the spin coating process. The process has been conducted for various spin speeds to change the centrifugal force, which influences the removed volume of the mixture in the micro holes. After that, concave menisci have been obtained in the holes because of the surface tension of the remaining mixture. Then, the heating process has been conducted to evaporate the solvent in the mixture and to solidify the photoresist so that deeper menisci could be obtained, which can be used as a mold for microlens array. The mixtures, which consist of photoresist and solvent, have been prepared in advance with different concentration. The fabrication method in this study is based on the volume shrinkage of the photoresist mixture, which is caused from the spin coating and evaporation processes. Therefore, it has been investigated that the geometry of the microlens can be modulated by the concentration of the mixture, as well as the process conditions of the spin coating and heating.
\end{abstract}

\section{Introduction}

As the one of the most fundamental components of the optical systems, the microlens array has been spotlighted, in order to improve the light-collection efficiency. Many optical devices are integrated with microlens array, such as charged-coupled device (CCD) array, digital integral photography, solar-cell, ultraviolet light-emitting diodes (UVLEDs) and so on. ${ }^{1-5}$ For fabrication of various kinds of the microlens arrays which satisfy specific requirements of each purpose, many researchers have been developing the fabrication processes of the micro-structures using diverse methods and materials. ${ }^{6-9}$ One of the conventional methods is the thermal reflow process. ${ }^{1,7,10}$ The reflow process consists of several steps as follows. At first, circular pillar structures, which are usually photosensitive materials, are patterned on the substrate through photo-lithography process. Then, the heating process is conducted to melt them, so that they are formed into the hemispheres due to the surface tension. Another well-known method is the laser machining process. Some studies have used volume expansion of the substrate by exposing it to the laser beam which is under the threshold energy of substrate. ${ }^{11,12}$ Other researchers have reported some results combining electrochemical techniques with laser machining to improve surface roughness. ${ }^{13}$ Ultraviolet (UV) printing methods, which usually use the negative photoresist with ultraviolet light source, are also candidates for fabrication of the microlens array. They include the ultraviolet proximity printing and tilted-exposure method. ${ }^{14,15}$ The ultraviolet proximity printing method uses the distribution of the light intensity caused by diffraction of the light at the mask. And the tiltedexposure method uses double irradiation of ultraviolet light in different angle so that the overlap regions become out-of-plane microlens. Nano imprint lithography is also a promising method for fabrication of microlens array which are smaller than $10 \mu \mathrm{m}$. Cheng et al. directly fabricated microlens array on the glass substrate using thermal nano imprinting with cylindrical molds. ${ }^{16}$ Other researchers have focused on electro-wetting method, which depends on the change of wettability through electrical modulation of the substrate. ${ }^{17} \mathrm{~S}$. Grilli et al. fabricated the liquid lenses using lithium niobate substrate, and its 
profile could be modulated actively by electrical signals. ${ }^{18}$ Another study, conducted by same group above, is about the direct fabrication process using liquid state of polydimethylsiloxane (PDMS). The profiles of the liquid PDMS droplets, which are dispensed on the conductive substrate, have been changed according to the wettability of the substrate which has been modulated by electric charges. ${ }^{19}$ Digital grayscale maskless lithography method consists of negative photosensitive substance and the micro-mirror device, which is controlled precisely by computer. By controlling the angle of each micro mirror, the light intensity to the negative photosensitive layer could be adjusted. ${ }^{20}$ When the vapor is condensed on liquid surface with the appropriate condition, the droplets are formed into the regular patterns, such as the honeycombs. This phenomenon is called as breath figures (BFs) method. It has been presented that the mold for the microlens array can be obtained using BFs method. ${ }^{21,22}$ Besides, D. Chandra et al. have applied ultraviolet ozone (UVO) treatment to the flatwise stretched PDMS. Then, the UVO treatment has caused partial volume expansion of the PDMS substrate so that convex microlens array could be fabricated. ${ }^{23}$

The curved surfaces can be also obtained when the liquid layer is coated on the micro structures due to its surface tension. This technique has been applied to the rectangular channels in order to obtain wavy patterns with smooth surfaces. ${ }^{24}$ The same technique can be used to make the mold for microlenses if the liquid layer is applied in the round holes, which results in the concave menisci. This can be done with the spin coating process ${ }^{25,26}$ or the evaporation of the liquid layer. ${ }^{27}$ In this study, we have coated photoresist mixture with the spin coater on the silicon substrate, where micro holes had been fabricated in advance, and then the mixture has been evaporated on the hot plate. Therefore, the concave menisci in the holes, which will be the profile of the microlens, have been modulated by the centrifugal force as well as the evaporation rate. The primary concave menisci have been obtained in the micro holes during the spin coating process. Then, the volatile components in the photoresist mixture have been evaporated during the soft baking process. The concave menisci, fabricated by the above two principles, have been replicated on the PDMS layer, so that we could obtain the microlens array.

\section{Fabrication Process}

The schematic of the fabrication process in this study is shown in Fig. 1. At first, deep reactive ion etching (DRIE) process has been conducted on the silicon substrate in order to make the micro holes. Although all the holes are $16 \mu \mathrm{m}$ deep, they have various diameters of $10,12,15$ and $20 \mu \mathrm{m}$ so that the influence of the geometry on the formation of menisci could be investigated. And the photoresist mixture has been prepared by mixing the photoresist (AZ-P4620, AZ Electronic Materials PLC.) and the solvent (AZ-1500K, AZ Electronic Materials PLC.). Then the mixture has been dispensed on the substrate which has the holes (Fig. 1(a)). The mixtures with different concentration ( 93 and $97 \mathrm{wt} \%$ of photoresist) have been used in order to change the viscosity of the mixture, which influences the spin coating process, and the amount of solvent, which influences the evaporating process. During the spin coating process, certain amount of

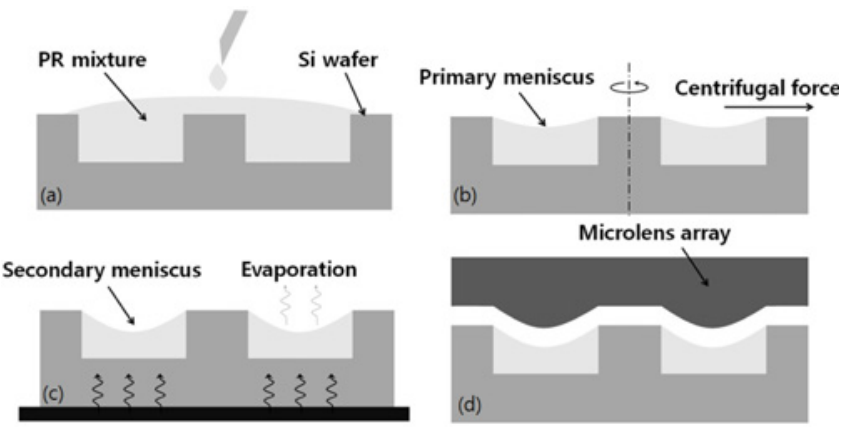

Fig. 1 Schematic of the fabrication process for the microlens array; (a) Dispensing of photoresist mixture on the silicon substrate with circular micro holes (b) Formation of the primary meniscus by centrifugal force during spin coating process (c) Formation of the secondary meniscus by evaporation of solvent during heating process (d) Replicating of the microlens array

the mixture has been removed from the micro holes by centrifugal force. Then, the remaining mixture in the holes have been formed into the concave menisci because of the surface tension and capillary phenomenon (Fig. 1(b)). Tests have been done for various spin speeds of 2000, 3000 and $4000 \mathrm{rpm}$ to change the centrifugal force. And then, heating process has been conducted at $80^{\circ} \mathrm{C}$ for $6 \mathrm{~min}$ to evaporate the solvent in the mixture so that the mixture has been shrunk and solidified (Fig. 1(c)). These secondary menisci could be used as a mold for microlens array. Polydimethylsiloxane (PDMS, Sylgard 184, Dow Corning Co.) and commercial replica polymer (RepliSet-F5, Struers Ltd.) have been used for replication of the microlens array (Fig. 1(d)). Then, the profile and height have been measured by scanning electron microscope (SEM, SM-350, TOPCON Co.) and interferometric microscope (Nano Surface Profiler, Nanosystem Co.), respectively.

\section{Measurements and Discussion}

Figs. 2 and 3 are the pictures of the microlens array replicated on the PDMS layer. They show that the microlens array has smooth and continuous surface with few defects. This is one of the advantages of this study because the mold is fabricated using liquid layer. Meanwhile, conventional processes, such as mechanical machining and chemical etching, usually need further treatment to improve the surface roughness. Therefore, it is expected that this fabrication process will produce the surfaces with fine optical quality without further process or cost.

Since the diameters of the lenses are fixed in this study, which are $10,12,15$ and $20 \mu \mathrm{m}$, the heights have been measured with an interferometric microscope At least 15 lenses have been measured and the averaged values have been obtained. As seen in Fig. 4, the height of the microlens is proportional to the spin speed. It is because the higher spin speed accompanies larger centrifugal force, which removes more amount of the photoresist mixture from the micro holes. It can be also noticed that the height of microlens is lower in smaller diameter with the same spin speed and concentration. Major driving forces to make the meniscus are the surface tension and the centrifugal force. The centrifugal force causes the removal of the photoresist mixture in 


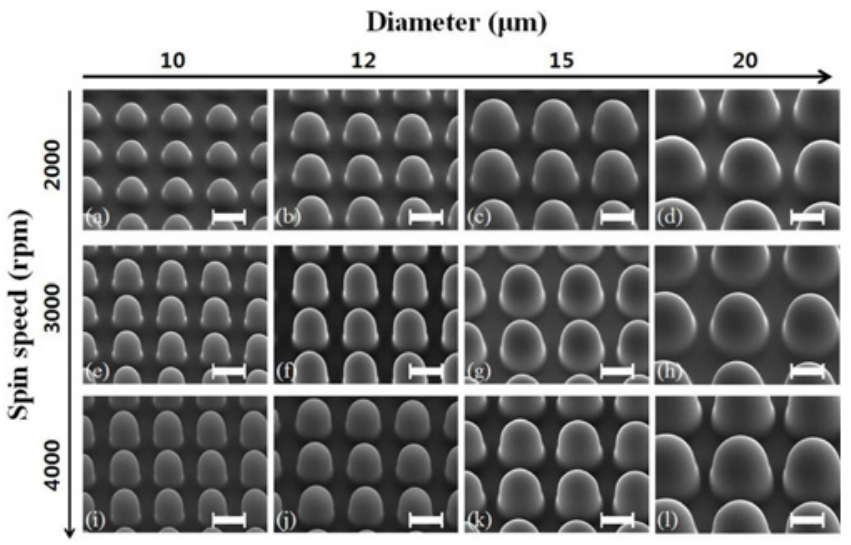

Fig. 2 SEM images of microlens array using mixture of $93 \mathrm{wt} \%$ of photoresist with various spin speeds $(2000,3000$, and $4000 \mathrm{rpm})$ and hole diameters $(10,12,15$, and $20 \mu \mathrm{m})$ (Scale bar: $10 \mu \mathrm{m})$

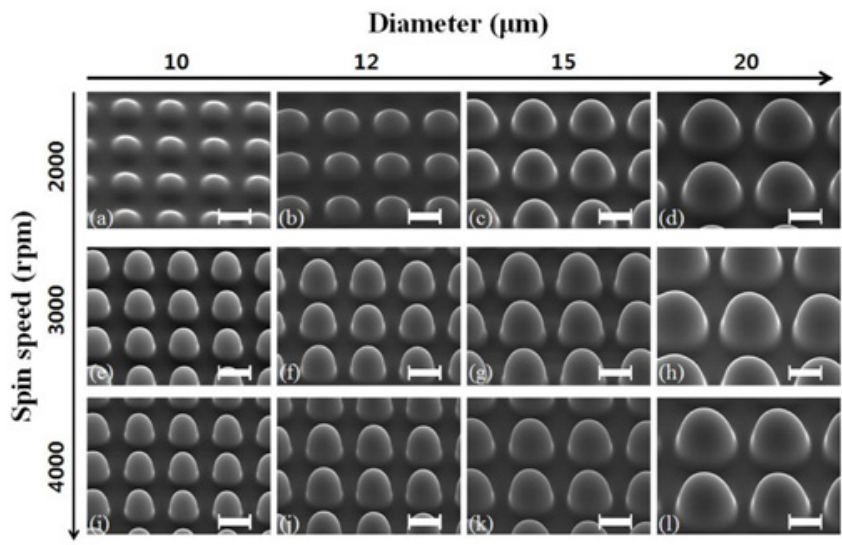

Fig. 3 SEM images of microlens array using mixture of $97 \mathrm{wt} \%$ of photoresist mixture with various spin speeds $(2000,3000$, and 4000 $\mathrm{rpm})$ and hole diameters (10, 12, 15, and $20 \mu \mathrm{m})$ (Scale bar: $10 \mu \mathrm{m})$

the micro holes, while the surface tension restrains it. Since the body force is more dominant in larger scale, which is the centrifugal force in this study, the height has become higher in the larger diameter. However, surface force gets more dominant in smaller scale, which is the surface tension, so the height has become smaller in the smaller diameter.

This means that the higher spin speed is needed to obtain a microlens with certain height if the diameter is smaller.

Fig. 4 also shows that the increasing rate of the height is not proportional but saturated to the spin speeds. The height of the microlens in this study depends on the remaining amount of the photoresist mixture in the micro holes. So its variation could be inferred from the basic principle of the spin coating process. ${ }^{28}$

$$
T=\frac{K C^{\beta} \eta^{\gamma}}{\omega^{\alpha}}
$$

where $T$ is the thickness of photoresist, $K$ is a calibration constant, $C$ is the polymer concentration, $\eta$ is viscosity and $\omega$ is the revolution speed (rpm). So the remaining mixture in the micro holes has decreased but stagnated as the spin speed increases, just like the thickness in the above equation. This means that the geometry of the microlens could be modulated by the spin speed, but it is only available under certain

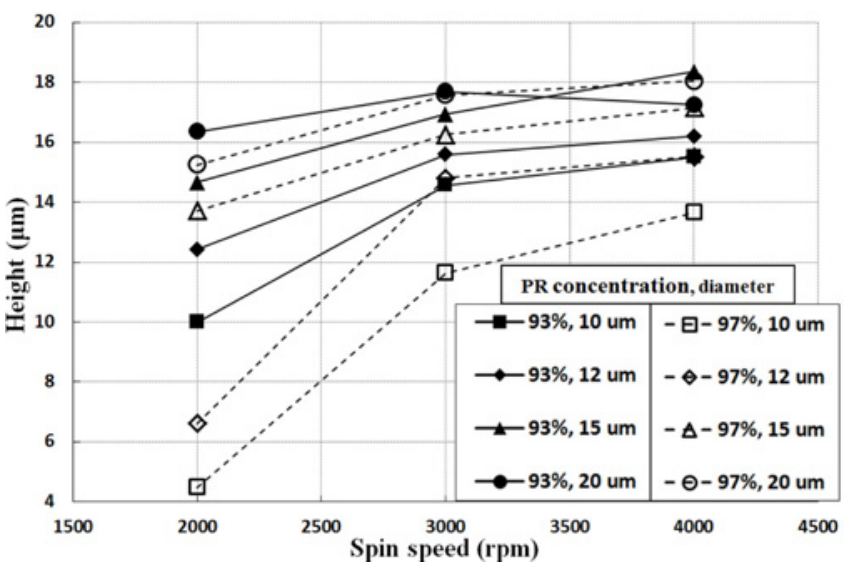

Fig. 4 Variation of heights of the microlens array for various diameters, photoresist concentrations in the mixtures, and spin speeds

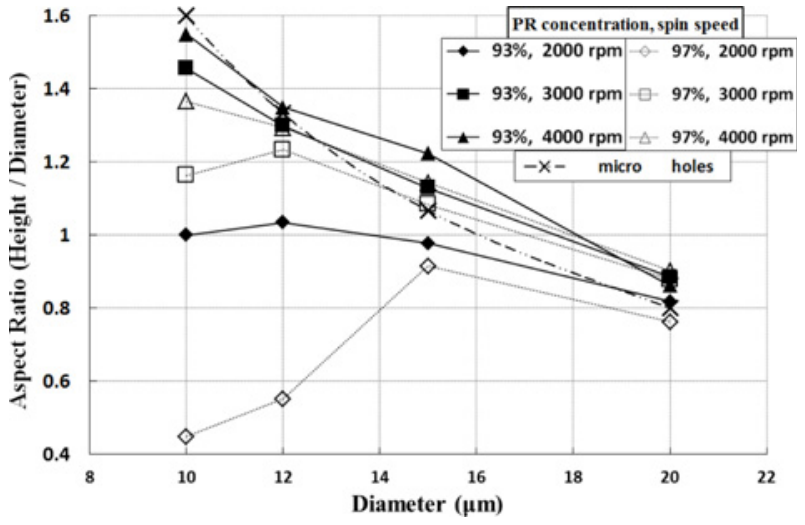

Fig. 5 Variation of aspect ratios of the microlens array in various diameters, photoresist concentrations in the mixtures, and spin speeds

range of the spin speed. Therefore, this study includes the variation of the concentration of the photoresist. The variation of the concentration is expected to have two influences on this process, which are the change of the viscosity of the mixture during the spin coating process, and the change of the evaporated volume of the mixture during the baking process. The viscosity of the mixture becomes smaller for lower concentration, which has made the primary meniscus deeper during the spin coating process even though other conditions have not been changed according to the above equation. Moreover, if concentration is smaller, more amount of the mixture is evaporated, which causes into the taller microlenses eventually. From this reason, the height variation is much sensitive to the change of the concentration as seen in Fig. 4. Therefore, the test results show that the change of the concentration can be used as another efficient method to modulate the geometry of the microlens.

For further investigation, the aspect ratio of the microlens has been calculated and illustrated as seen in Fig. 5. Since all of the micro holes have the same depths with various diameters, the aspect ratios of the micro holes are inversely proportional to the diameters. Accordingly, the aspect ratios of the microlenses tend to decrease with the diameters overall. However, it is remarkable that the aspect ratios are very diverse when the diameters are small (10 and $12 \mu \mathrm{m})$, while they tend to converge when the diameters are larger (15 and $20 \mu \mathrm{m})$. Moreover, the aspect ratios are more scattered if the spin speed is lower. Accordingly, 
Table 1 Standard deviations of the measured heights of the microlenses

\begin{tabular}{|c|c|c|c|}
\hline \multirow[b]{2}{*}{$\begin{array}{l}\text { Diameter } \\
(\mu \mathrm{m})\end{array}$} & \multicolumn{3}{|c|}{ Standard deciations $(\mu \mathrm{m})$} \\
\hline & $\begin{array}{l}\text { Spin } \\
\text { speed } \\
(\mathrm{rpm})\end{array}$ & $\begin{array}{c}\text { Photoresist } \\
\text { concentration } \\
93 \% \\
\end{array}$ & $\begin{array}{c}\text { Photoresist } \\
\text { concentration } \\
97 \% \\
\end{array}$ \\
\hline \multirow{3}{*}{10} & 2000 & 0.771487 & 0.200327 \\
\hline & 3000 & 0.170315 & 0.242271 \\
\hline & 4000 & 0.140946 & 0.145979 \\
\hline \multirow{3}{*}{12} & 2000 & 0.392969 & 0.387472 \\
\hline & 3000 & 0.219430 & 0.142625 \\
\hline & 4000 & 0.149548 & 0.057310 \\
\hline \multirow{3}{*}{15} & 2000 & 0.396144 & 0.242779 \\
\hline & 3000 & 0.118142 & 0.119246 \\
\hline & 4000 & 0.184254 & 0.168774 \\
\hline \multirow{3}{*}{20} & 2000 & 0.455725 & 0.110249 \\
\hline & 3000 & 0.258987 & 0.216229 \\
\hline & 4000 & 0.095376 & 0.181532 \\
\hline
\end{tabular}

the largest difference has been obtained from the results between $93 \%$ and $97 \%$ for $2000 \mathrm{rpm}$ in $10 \mu \mathrm{m}$ holes. Since the surface tension plays an important role in the small diameter, the change of the concentration, which causes into the change of the surface tension, has influenced much on the results, as well as the spin speed. So it can be stated that the geometry of the microlens could be modulated in wide range, if the concentration and spin speed are adjusted properly especially when the diameter is small. It is also noticeable that the variations of the aspect ratios in the larger diameters are nearly parallel to each other, in accordance with those of the micro holes. Since the surface tension is not so influential here, the volume change of the photoresist mixture could be assumed to depend majorly on the concentration and the spin speed. Therefore, this result is expected to be used as a process map in the future works to provide fabrication conditions for a target geometry, considering the material's property also.

\section{Conclusions}

In this study, a micro mold for the microlens array has been fabricated using spin coating and heating processes with photoresist mixture, which has different concentration of photoresist. There are two major parameters to modulate the menisci in the micro holes, which are the geometry of the mold for microlenses. The first one is the centrifugal force applied by spin coating process, which could be controlled by manipulating the spin speed. Since higher spin speed means larger centrifugal force, the height of the microlens could be increased with the spin speed. The second one is the surface tension and viscosity of the photoresist mixture, which prevent removal of the mixture from the hole. Because those properties could be modified by using different composition of photoresist and solvent, the geometry of the microlens has been modulated also. So it has been investigated in this study that the geometry of the microlens array could be defined according to concentration of photoresist mixture, spin speed and diameter of the holes. And it is also expected that the evaporation speed is another parameter to modulate the geometry of the microlenses. So the future works need to include the investigation on the evaporation speed. The fabrication process presented in this study is quite simple and post process or treatment is not required. Furthermore, the fabrication method in this study has quite good uniformity as seen in Table 1. So this study is expected to provide a process method which is cost effective and environmentally friendly for a lot of applications such as solar cells and micro optical devices.

\section{ACKNOWLEDGEMENT}

This study was supported by the Industrial Strategic technology development program (No.10042797, Development of master machining system and $10 \%$ energy saving molding system for $100 \mathrm{~nm} \sim 100 \mu \mathrm{m}$ nano hybrid structures) funded by the Ministry of Trade, Industry and Energy of Korea and the Institute Project (NK188B).

\section{REFERENCES}

1. Nussbaum, P., Voelkel, R., Herzig, H. P., Eisner, M., and Haselbeck, S., "Design, Fabrication and Testing of Microlens Arrays for Sensors and Microsystems," Pure and Applied Optics: Journal of the European Optical Society Part A, Vol. 6, No. 6, pp. 617-636, 1997.

2. Chronis, N., Liu, G., Jeong, K.-H., and Lee, L., "Tunable LiquidFilled Microlens Array Integrated with Microfluidic Network," Optics Express, Vol. 11, No. 19, pp. 2370-2378, 2003.

3. Erdmann, L. and Gabriel, K. J., "High-Resolution Digital Integral Photography by Use of a Scanning Microlens Array," Applied Optics, Vol. 40, No. 31, pp. 5592-5599, 2001.

4. Tseng, J., Chen, Y., Pan, C., Wu, T., and Chung, M., “Application of Optical Film with Micro-Lens Array on a Solar Concentrator," Solar Energy, Vol. 85, No. 9, pp. 2167-2178, 2011.

5. Khizar, M., Fan, Z., Kim, K., Lin, J., and Jiang, H., "Nitride DeepUltraviolet Light-Emitting Diodes with Microlens Array," Applied Physics Letters, Vol. 86, No. 17, Paper No. 173504, 2005.

6. Chu, W.-S., Kim, C.-S., Lee, H.-T., Choi, J.-O., Park, J.-I., et al., "Hybrid Manufacturing in Micro/Nano Scale: A Review," Int. J. Precis. Eng. Manuf.-Green Tech., Vol. 1, No. 1, pp. 75-92, 2014.

7. Lian, Z.-J., Hung, S.-Y., Shen, M.-H., and Yang, H., "Rapid Fabrication of Semiellipsoid Microlens Using Thermal Reflow with Two Different Photoresists," Microelectronic Engineering, Vol. 115, pp. 46-50, 2014.

8. Kang, D. J. and Bae, B.-S., "Photo-Imageable Sol-Gel Hybrid Materials for Simple Fabrication of Micro-Optical Elements," Accounts of Chemical Research, Vol. 40, No. 9, pp. 903-912, 2007.

9. Zhang, X., Que, W., Javed, H. M. A., and Wei, W., "Elliptical Concave Microlens Arrays Built in the Photosensitive TiO 2/ Ormosils Hybrid Films," Optics Communications, Vol. 330, pp. 1218, 2014. 
10. O’Neill, F. T. and Sheridan, J. T., "Photoresist Reflow Method of Microlens Production, Part I: Background and Experiments," Optik-International Journal for Light and Electron Optics, Vol. 113, No. 9, pp. 391-404, 2002.

11. Kim, K.-R., Jeong, H.-W., Lee, K.-S., Yi, J., Yoo, J.-C., et al., "Rapid Laser Fabrication of Microlens Array Using Colorless Liquid Photopolymer for AMOLED Devices," Optics Communications, Vol. 284, No. 1, pp. 405-410, 2011.

12. Je, S., Park, S., Choi, C., and Shin, B., "A Study on Rapid Fabrication of Micro Lens Array Using $355 \mathrm{~nm}$ UV Laser Irradiation," Transactions of Materials Processing, Vol. 18, No. 4, pp. 310-316, 2009.

13. Kikuchi, T., Wachi, Y., Takahashi, T.-A., Sakairi, M., and Suzuki, R. O., "Fabrication of a Meniscus Microlens Array Made of Anodic Alumina by Laser Irradiation and Electrochemical Techniques," Electrochimica Acta, Vol. 94, pp. 269-276, 2013.

14. Lin, C. P., Yang, H., and Chao, C. K., "A New Microlens Array Fabrication Method Using UV Proximity Printing," Journal of Micromechanics and Microengineering, Vol. 13, No. 5, pp. 748-757, 2003.

15. Zhang, Y., Cui, M., Fan, X., Zhang, B., and Wang, W., "The Fabrication and Fast Replication of Out of Plane Parabolic Microlens Arrays," Sensors and Actuators A: Physical, Vol. 216, pp. 190-195, 2014.

16. Cheng, M. C., Chen, L. K., and Sung, C. K., "Fabrication of Controllable Profile Micrllens Array by Nanoimprinting Process," Proc. of the $7^{\text {th }}$ IEEE International Conference on Nano/Micro Engineered and Molecular Systems, pp. 542-546, 2012.

17. Zeng, X. and Jiang, H., "Liquid Tunable Microlenses Based on MEMS Techniques," Journal of Physics D: Applied Physics, Vol. 46, No. 32, Paper No. 323001, 2013.

18. Grilli, S., Miccio, L., Vespini, V., Finizio, A., Nicola, S., et al., "Liquid Micro-Lens Array Activated by Selective Electrowetting on Lithium Niobate Substrates," Optics Express, Vol. 16, No. 11, pp. 8084-8093, 2008.

19. Grilli, S., Vespini, V., and Farraro, P., "Surface-Charge Lithography for Direct PDMS Micro-Patterning," Langmuir, Vol. 24, No. 23, pp. 13262-13265, 2008.

20. Zhong, K., Gao, Y., Li, F., Zhang, Z., and Lui, N., "Fabrication of PDMS Microlens Array by Digital Maskless Grayscale Lithography and Replica Molding Technique," Optik-International Journal for Light and Electron Optics, Vol. 125, No. 10, pp. 2413-2416, 2014.

21. Escalé, P., Rubatat, L., Billon, L., and Save, M., "Recent Advances in Honeycomb-Structured Porous Polymer Films Prepared via Breath Figures," European Polymer Journal, Vol. 48, No. 6, pp. 1001-1025, 2012.

22. Wu, C. Y., Chiang, T. H., and Hsu, C. C., "Fabrication of Microlens Array Diffuser Films with Controllable Haze Distribution by Combination of Breath Figures and Replica Molding Methods," Optics Express, Vol. 16, No. 24, pp. 19978-19986, 2008.
23. Chandra, D., Yang, S., and Lin, P.-C., "Strain Responsive Concave and Convex Microlens Arrays," Applied Physics Letters, Vol. 91, No. 25, Paper No. 251912, 2007.

24. Yoon, J. S., Yoo, Y. E., and Choi, D. S., "A Study on the Fabrication of Rounded Patterns by Spin Coating of Photoresist on Silicon Substrate with Microstructures," Polymer Engineering and Science, Vol. 52, No. 3, pp. 499-506, 2012.

25. Shih, T. K., Chen, C. F., Ho, J. R., and Chuang, F. T., "Fabrication of Various Curved Relief Structures through Concave Surface Forming and Soft Replica Molding," Microelectronic Engineering, Vol. 83, No. 3, pp. 471-475, 2006.

26. Shih, T. K., Chen, C. F., Ho, J. R., and Chuang, F. T., "Fabrication of PDMS (polydimethylsiloxane) Microlens and Diffuser Using Replica Molding," Microelectronic Engineering, Vol. 83, No. 11, pp. 2499-2503, 2006.

27. Yoon, J. S., Lim, S. H., Kim, J. H., Yoo, Y. E., and Choi, D. S., “A Study on the Fabrication of Microlens Array Based on the Volume Shrinkage of the Photoresist Solution during Evaporation," Optics Communications, Vol. 332, pp. 70-74, 2014.

28. Madou, M., "Fundamentals of Microfabrication," CRC Press, 1997. 